\title{
Logic-Based Switching Control for Trajectory-Tracking and Path-Following of Underactuated Autonomous Vehicles with Parametric Modeling Uncertainty
}

\author{
António Pedro Aguiar and João Pedro Hespanha
}

\begin{abstract}
We address the problem of trajectory-tracking and path-following control design for underactuated autonomous vehicles in the presence of possibly large modeling parametric uncertainty. For a general class of vehicles moving in either two or three-dimensional space, we demonstrate how adaptive switching supervisory control can be combined with a nonlinear Lyapunov-based tracking control law to solve the problem of global boundedness and convergence of the position tracking error to a neighborhood of the origin that can be made arbitrarily small. We also show how these results can be extended to the path-following problem, in which the vehicle is required to converge to and follow a path, without a special temporal specification, and once in the path, to satisfy a desired dynamic behavior. We illustrate our design procedures through two vehicle control applications: a hovercraft (moving on a planar surface) and an underwater vehicle (moving in three-dimensional space). Simulations results are presented and discussed.
\end{abstract}

\section{INTRODUCTION}

The classical approach for trajectory-tracking of underactuated vehicles utilizes local linearization and decoupling of the multi-variable model to steer the same number of degrees of freedom as the number of available control inputs, which can be done using standard linear (or nonlinear) control methods. Alternative approaches include the linearization of the vehicle error dynamics around trajectories that lead to a time-invariant linear system (also known as trimming trajectories) combined with gain scheduling and/or Linear Parameter Varying (LPV) design methodologies [1]. The basic limitation of these approaches is that stability is only guaranteed in a neighborhood of the selected operating points. Moreover, performance can suffer significantly when the vehicle executes maneuvers that emphasize its nonlinearity and cross-couplings.

Nonlinear Lyapunov-based designs can overcome some of the limitations mentioned above. Several examples of nonlinear trajectory-tracking controllers for marine underactuated vehicles have been reported in the literature [2][5]. Typically, tracking problems for autonomous vehicles are solved by designing control laws that make the vehicles track pre-specified feasible "state-space" trajectories, i.e., trajectories that specify the time evolution of the position, orientation, as well as the linear and angular velocities,

This material is based upon work supported by the National Science Foundation under Grant No. ECS-0093762. The work of A. Pedro Aguiar was supported by a Pos-Doc Fellowship PRAXIS XXI from the Portuguese Foundation of Science and Technology

A. Pedro Aguiar and João P. Hespanha are with Department of Electrical and Computer Engineering, Mathematics and Computer Science, University of California, Santa Barbara, CA 93106-9560, USA \{aguiar, hespanha\} @ece.ucsb.edu all consistent with the vehicles' dynamics, [2]-[5], even through in practical applications one often only needs to track a desired position. This approach suffers from the drawback that usually the vehicles' dynamics exhibit complex nonlinear terms and significant uncertainty, which makes the task of computing a feasible trajectory difficult.

Motivated by the above considerations, in [6] we proposed a solution to the position tracking problem for a fairly general class of underactuated autonomous vehicles that is applicable to motion in either two or three dimensional spaces. The desired trajectory does not need to be a trimming trajectory and can be any sufficiently smooth time-varying bounded curve, including the degenerate case of a constant trajectory (set-point).

To address parametric uncertainty in the process model, in this paper (Section III) we show how supervisory control can be combined with the design procedure outlined in [6]. The basic idea behind supervisory control [7]-[11] is to design a suitable family of candidate controllers. Each controller is designed for an admissible nominal model of the process, and a supervision logic orchestrates the switching among the candidate controllers, deciding, at each instant of time, the candidate feedback controller that is more adequate. In order to guarantee stability and avoid chattering, a form of hysteresis is employed. In [9], logic-based switching control laws were derived to park a nonholonomic wheeled mobile robot with parameter uncertainty.

A distinct class of motion control problems for autonomous vehicles is path-following. In path-following, the vehicle is required to converge to and follow a path that is specified without a temporal law [12]-[14]. Pioneering work in this area for wheeled mobile robots is described in [12]. In [13], Samson addressed the path-following problem for a car pulling several trailers. Path-following controllers for aircraft and marine vehicles have been reported in [1], [15]-[17]. Using the approach suggested by Hauser and Hindman [15], an output maneuvering controller was proposed in [18] for a class of strict feedback nonlinear processes and applied to path-following of fully actuated ships. The underlying assumption in path-following is that the vehicle's forward speed tracks a desired speed profile, while the controller acts on the vehicle's orientation to drive it to the path. Typically, in path-following, smoother convergence to the path is achieved and the control signals are less likely pushed into saturation, when compared to trajectory-tracking [15], [16]. In fact, in [19] we highlight a fundamental difference between path-following and stan- 
dard trajectory-tracking by demonstrating that performance limitations due to unstable zero-dynamics can be removed in the path-following problem.

Inspired by these ideas, in Section IV, we re-design the tracking controller to solve the path-following problem by decomposing it into two subproblems: i) a geometric task, which consists of converging the vehicle to and remaining inside a tube centered around the desired path, and ii) a dynamic assignment task, which assigns a speed profile to the path. In Section V, we illustrate our design methodologies in the context of two vehicle control applications: a hovercraft (moving on a planar surface) and an underwater vehicle (moving in three-dimensional space). The designs are validated through computer simulations. The paper concludes with a summary of the results and suggestions for further research.

Due to space limitations, all the proofs are omitted. These can be found in [20].

\section{Problem Statement}

Consider an underactuated vehicle modeled as a rigid body subject to external forces and torques. Let $\{\mathcal{I}\}$ be an inertial coordinate frame and $\{B\}$ a body-fixed coordinate frame whose origin is located at the center of mass of the vehicle. The configuration $(R, p)$ of the vehicle is an element of the Special Euclidean group $S E(3):=$ $S O(3) \times \mathbb{R}^{3}$, where $R \in S O(3):=\left\{R \in \mathbb{R}^{3 \times 3}: R R^{\prime}=\right.$ $\left.I_{3}, \operatorname{det}(R)=+1\right\}$ is a rotation matrix that describes the orientation of the vehicle by mapping body coordinates into inertial coordinates, and $p \in \mathbb{R}^{3}$ is the position of the origin of $\{B\}$ in $\{\mathcal{I}\}$. Denoting by $v \in \mathbb{R}^{3}$ and $\omega \in \mathbb{R}^{3}$ the linear and angular velocities of the vehicle relative to $\{\mathcal{I}\}$ expressed in $\{B\}$, respectively, the following kinematic relations apply

$$
\begin{aligned}
\dot{p} & =R v, \\
\dot{R} & =R S(\omega),
\end{aligned}
$$

where $S(x):=\left[\begin{array}{ccc}0 & -x_{3} & x_{2} \\ x_{3} & 0 & -x_{1} \\ -x_{2} & x_{1} & 0\end{array}\right], \forall x:=\left(x_{1}, x_{2}, x_{3}\right) \in \mathbb{R}^{3}$. We consider here underactuated vehicles with dynamic equations of motion of the following form:

$$
\begin{aligned}
\mathbf{M} \dot{v} & =-S(\omega) \mathbf{M} v+f_{v}(v, \omega, R)+g_{1} u_{1} \\
\mathbf{J} \dot{\omega} & =-S(v) \mathbf{M} v-S(\omega) \mathbf{J} \omega+f_{\omega}(v, \omega, R)+G_{2} u_{2}
\end{aligned}
$$

where $\mathbf{M} \in \mathbb{R}^{3 \times 3}$ and $\mathbf{J} \in \mathbb{R}^{3 \times 3}$ denote constant symmetric positive definite mass and inertia matrices; $u_{1} \in \mathbb{R}$ and $u_{2} \in \mathbb{R}^{3}$ denote the control inputs, which act upon the system through a constant nonzero vector $g_{1} \in \mathbb{R}^{3}$ and a constant nonsingular matrix ${ }^{1} G_{2} \in \mathbb{R}^{3 \times 3}$, respectively; and $f_{v}(v, \omega, R), f_{\omega}(v, \omega, R)$ represent all the remaining forces and torques acting on the body. For the special case of an underwater vehicle, $\mathbf{M}$ and $\mathbf{J}$ also include the so-called hydrodynamic added-mass $M_{A}$ and addedinertia $J_{A}$ matrices, respectively, i.e., $\mathbf{M}=M_{R B}+M_{A}$, $\mathbf{J}=J_{R B}+J_{A}$, where $M_{R B}$ and $J_{R B}$ are the rigid-body mass and inertia matrices, respectively.

\footnotetext{
${ }^{1}$ The special case of $G_{2} \in \mathbb{R}^{3 \times 2}, u_{2} \in \mathbb{R}^{2}$ can also be addressed. Details can be found in [20].
}

For an underactuated vehicle restricted to move on a planar surface, the same equations of motion (1)-(2) apply without the first two right-hand side terms in (2b). Also, in this case, $(R, p) \in S E(2), \nu \in \mathbb{R}^{2}, \omega \in \mathbb{R}, g_{1} \in \mathbb{R}^{2}$, $G_{2} \in \mathbb{R}, u_{2} \in \mathbb{R}$, with all the other terms in (2) having appropriate dimensions, and the skew-symmetric matrix $S(\omega)$ is given by $S(\omega)=\left(\begin{array}{cc}0 & -\omega \\ \omega & 0\end{array}\right)$. For simplicity, in what follows, we restrict our attention to the three-dimensional case. However, all results are directly applicable to the twodimensional case, as will be illustrated in Section V-A for the control of a Hovercraft.

The following technical assumptions are made:

Assumption 1: The function $f_{v}(v, \omega, R)$ is affine on $\omega$, i.e., $f_{v}(v, \omega, R)=f_{v_{1}}(v, R)+f_{v_{2}}(v, R) \omega, \quad \forall v, \omega \in$ $\mathbb{R}^{3}, R \in S O(3)$.

Assumption 2: There exists a vector $\delta \in \mathbb{R}^{3}$ such that for every $t \geq 0, v \in \mathbb{R}^{3}, R \in S O(3)$, the following matrix is full-rank

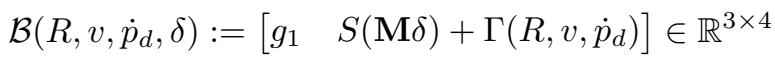

where $\Gamma\left(R, v, \dot{p}_{d}\right):=S\left(\mathbf{M} R^{\prime} \dot{p}_{d}\right)-\mathbf{M} S\left(R^{\prime} \dot{p}_{d}\right)+f_{v_{2}}(v, R)$.

These assumptions seem to hold for a large class of vehicle models, which includes the Hovercraft and the underwater vehicles discussed in Section V.

The problems considered in this paper can be stated as follows:

Trajectory-tracking problem: Let $p_{d}(t):[0, \infty) \rightarrow \mathbb{R}^{3}$ be a given sufficiently smooth time-varying desired trajectory with its time-derivatives bounded. Design a controller such that all the closed-loop signals are bounded and the tracking error $\left\|p(t)-p_{d}(t)\right\|$ converges to a neighborhood of the origin that can be made arbitrarily small.

Path-following problem: Let $p_{d}(\gamma) \in \mathbb{R}^{3}$ be a desired path parameterized by a continuous variable $\gamma \in \mathbb{R}$ and $v_{r}(\gamma) \in \mathbb{R}$ a desired speed ${ }^{2}$ assignment. Suppose also that $p_{d}(\gamma)$ is sufficiently smooth and its derivatives (with respect to $\gamma)$ are bounded. Design a controller such that all the closed-loop signals are bounded, and the position of the vehicle i) converges to and remains inside a tube centered around the desired path that can be made arbitrarily thin, i.e., $\left\|p(t)-p_{d}(\gamma(t))\right\|$ converges to a neighborhood of the origin that can be made arbitrarily small, and ii) satisfies a desired speed assignment $v_{r}$ along the path, i.e., $\mid \dot{\gamma}(t)-$ $v_{r}(\gamma(t)) \mid \rightarrow 0$ as $t \rightarrow \infty$.

\section{Trajectory-Tracking CONTROLler Design}

This section proposes an estimator based supervisory control architecture to solve the trajectory-tracking problem in the presence of parametric modeling uncertainty.

Let $\Theta \in \mathbb{R}^{n_{\Theta}}$ be a vector that contains all the unknown parameters of the dynamic equations of motion (2), where $n_{\Theta}$ denotes the number of unknown parameters. The following technical assumption is assumed to hold:

\footnotetext{
${ }^{2}$ For simplicity of presentation it will be assumed that the speed assignment $v_{r}(\gamma) \in \mathbb{R}$ does not depend directly on time $t$.
} 


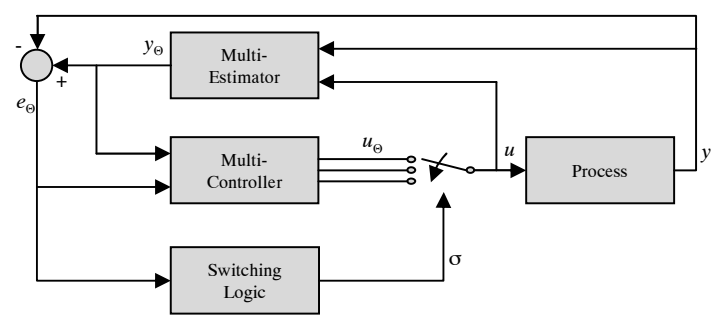

Fig. 1. Supervisory control architecture.

Assumption 3: Let $\mathcal{P}$ be a finite set of candidate parameter values $\mathcal{P}:=\left\{\Theta_{1}, \Theta_{2}, \ldots, \Theta_{N}\right\}$. The actual parameter $\Theta^{\star}$ belongs to $\mathcal{P}$.

In practice, this assumption can be relaxed to $\Theta^{\star}$ being sufficiently close to an element of $\mathcal{P}$, which can be achieved by taking a fine grid, at the price of increased computational burden.

The supervisory control architecture consists of tree subsystems (see Fig. 1) [9], [10]: the multi-estimator, whose inputs are the process input $u$ and its output $y$, and whose outputs are $y_{\Theta}, \Theta \in \mathcal{P}$, where each $y_{\Theta}$ is a suitably defined estimate of $y$ which would be asymptotically correct if $\Theta^{\star}$ was equal to $\Theta$; the multi-controller, whose inputs are the output estimate $y_{\Theta}$ and the estimation errors $\mathbf{e}_{\Theta}:=y_{\Theta}-y$, $\Theta \in \mathcal{P}$, and whose outputs are the control signals $u_{\Theta}$, $\Theta \in \mathcal{P}$, where each $u_{\Theta}$ is generated by a control law that would be adequate if $\Theta^{\star}$ was equal to $\Theta$; and the switching logic, whose inputs are the estimation errors $\mathbf{e}_{\Theta}$ and whose output is a switching signal $\sigma$ which is used to define the control law $u=u_{\sigma}$.

The underlying decision-making strategy used by the switching logic basically consists of selecting for $\sigma$, the candidate controller index $\Theta^{\dagger}$ for which the corresponding performance signal $\mu_{\Theta^{\dagger}}$ is currently the smallest. This strategy is motivated by the idea that the nominal process model with the smallest performance signal is the one that "best" approximates the actual process, and thus the candidate controller associated with that model can be expected to have a better performance of controlling the process.

In this paper we assume that the whole state of the process is available for feedback. Therefore, $y=(p, R, v, \omega)$, $y_{\Theta}=\left(p, R, v_{\Theta}, \omega_{\Theta}\right)$, and $\mathbf{e}_{\Theta}:=\hat{y}_{\Theta}-y=\left(\tilde{v}_{\Theta}, \tilde{\omega}_{\Theta}\right)$. Note, that since there is no uncertainty in (1), we can simply pick $p_{\Theta}=p$ and $R_{\Theta}=R$. We also restrict our attention to state feedback laws and therefore $u_{\Theta}=\mathcal{K}_{\Theta}\left(p, R, v, \omega, \tilde{v}_{\Theta}, \tilde{\omega}_{\Theta}\right)$.

\section{A. Multi-estimator}

This section addresses the design of a family of estimators parameterized by $\Theta \in \mathcal{P}$ for the underactuated vehicle model (1)-(2). Motivated by Assumption 3 and in view of (2), we consider a family of estimator equations of the form ${ }^{3}$

$$
\begin{aligned}
& \mathbf{M}_{\Theta} \dot{\hat{v}}_{\Theta}=-S(\omega) \mathbf{M}_{\Theta} v+f_{v_{1_{\Theta}}}(v, R)+f_{v_{2_{\Theta}}}(v, R) \omega \\
& +g_{1} u_{1}-\mathbf{M}_{\Theta} L_{v} \tilde{v}_{\Theta}-\beta_{v_{\Theta}}\left(v, \omega, \hat{v}_{\Theta}, R\right) \mathbf{M}_{\Theta} \tilde{v}_{\Theta}
\end{aligned}
$$

\footnotetext{
${ }^{3}$ When $\mathcal{P}$ has a large number of elements, an alternative approach, which leads to a more efficient design, is to replace the individual estimator equations by a single system and use it to generate the estimation errors, in other words, to make the estimators "share" the same state. See details in $[20]$
}

$$
\begin{array}{r}
\mathbf{J}_{\Theta} \dot{\hat{\omega}}_{\Theta}=-S(v) \mathbf{M}_{\Theta} v-S(\omega) \mathbf{J}_{\Theta} \omega+f_{\omega_{\Theta}}(v, \omega, R) \\
+G_{2_{\Theta}} u_{2}-\mathbf{J}_{\Theta} L_{\omega} \tilde{\omega}_{\Theta}-\beta_{\omega_{\Theta}}\left(\hat{v}_{\Theta}, \omega, \hat{\omega}_{\Theta}, R\right) \mathbf{J}_{\Theta} \tilde{\omega}_{\Theta}
\end{array}
$$

where $L_{v}, L_{\omega} \in \mathbb{R}^{3 \times 3}$ are diagonal positive definite matrices and for each $\Theta \in \mathcal{P}$ the scalar positive functions $\beta_{v_{\Theta}}:=\beta_{1_{\Theta}}+\beta_{3_{\Theta}}$ and $\beta_{\omega_{\Theta}}:=\beta_{2_{\Theta}}+\beta_{4_{\Theta}}$ satisfy

$$
\begin{gathered}
\left\|f_{v_{\Theta}}\left(\hat{v}_{\Theta}, R\right)-f_{v}(v, R)\right\|+\left\|f_{v_{\Theta}}\left(\hat{v}_{\Theta}, R\right) \omega-f_{v}(v, R) \omega\right\| \\
+\left\|S(\omega) M_{\Theta} \tilde{v}_{\Theta}\right\| \leq c_{1_{\Theta}} \beta_{1_{\Theta}}\left(v, \omega, \hat{v}_{\Theta}, R\right)\left\|\tilde{v}_{\Theta}\right\| \\
\left\|f_{v_{2_{\Theta}}}\left(\hat{v}_{\Theta}, R\right) \tilde{\omega}_{\Theta}\right\| \leq c_{2_{\Theta}} \beta_{2_{\Theta}}\left(\hat{v}_{\Theta}, R\right)\left\|\tilde{\omega}_{\Theta}\right\| \\
\left\|f_{\omega}\left(\hat{v}_{\Theta}, \omega, R\right)-f_{\omega}(v, \omega, R)\right\| \leq c_{3_{\Theta}} \beta_{3_{\Theta}}\left(v, \hat{v}_{\Theta}, \omega, R\right)\left\|\tilde{v}_{\Theta}\right\| \\
\left\|f_{\omega}\left(\hat{v}_{\Theta}, \hat{\omega}_{\Theta}, R\right)-f_{\omega}\left(\hat{v}_{\Theta}, \omega, R\right)\right\| \leq c_{4_{\Theta}} \beta_{\beta_{\Theta}}\left(\hat{v}_{\Theta}, \omega, \hat{\omega}_{\Theta}, R\right)\left\|\tilde{\omega}_{\Theta}\right\|
\end{gathered}
$$

for some positive constants $c_{i_{\Theta}}, i=1, \ldots, 4$. The multiestimator has the desirable property that the estimator error that corresponds to the true parameter value $\Theta^{\star}$ converges exponentially to zero and satisfies a $\mathcal{L}_{1}$-like property.

Lemma 1: Let $\Theta^{\star} \in \mathcal{P}$ be the actual parameter value. For every initial condition of equations (1b), (2), and (3), respectively, and continuous signal $u$, there exist positive constants $\kappa, \lambda_{\mu}, c_{1}, c_{2}, c_{3}$ such that

$$
\begin{gathered}
\left\|\mathbf{e}_{\Theta^{\star}}(t)\right\| \leq e^{-\kappa t} c_{1}, \\
\int_{0}^{t} e^{\lambda_{\mu} \tau} \beta_{v_{\Theta^{\star}}}\left(v(\tau), \omega(\tau), \hat{v}_{\Theta^{\star}}(\tau), R(\tau)\right)\left\|\tilde{v}_{\Theta^{\star}}(\tau)\right\| d \tau \leq c_{2}, \\
\int_{0}^{t} e^{\lambda_{\mu} \tau} \beta_{\omega_{\Theta^{\star}}}\left(\hat{v}_{\Theta}(\tau), \omega(\tau), \hat{\omega}_{\Theta}(\tau), R(\tau)\right)\left\|\tilde{\omega}_{\Theta^{\star}}(\tau)\right\| d \tau \leq c_{3},
\end{gathered}
$$

$\forall t \in[0, T)$ where $[0, T), T \in(0,+\infty]$ denotes the maximum interval of existence of solutions to the closedloop.

\section{B. Multi-controller}

We now design a family of candidate feedback laws $\mathcal{K}_{\Theta}(\cdot)$ such that for each $\Theta \in \mathcal{P}, u=\mathcal{K}_{\Theta}(\cdot)$ would solve the tracking problem formulated in Section II for a process model given by (1) and (3), and "sufficiently" small estimator errors $\tilde{v}_{\Theta}, \tilde{\omega}_{\Theta}$. For a given $\Theta \in \mathcal{P}$, we design $\mathcal{K}_{\Theta}$ by constructing control-Lyapunov functions iteratively, following the design procedure proposed in [6].

Step 1. Coordinate transformation: Consider the global diffeomorphic coordinate transformation $e:=R^{\prime}\left(p-p_{d}\right)$, which expresses the tracking error $p-p_{d}$ in the body-fixed frame. The dynamic equation of the tracking error $e$ is given by

$$
\dot{e}=-S(\omega) e+v-R^{\prime} \dot{p}_{d}
$$

Step 2. Convergence of $e$ : We start by defining the control-Lyapunov function $V_{1}:=\frac{1}{2} e^{\prime} e$ and computing its time derivative to obtain

$$
\dot{V}_{1}=e^{\prime}\left[\hat{v}_{\Theta}-\tilde{v}_{\Theta}-R^{\prime} \dot{p}_{d}\right] .
$$

We can regard $\hat{v}_{\Theta}$ as a virtual control that one would use to make $\dot{V}_{1}$ negative. Assuming that $\tilde{v}_{\Theta}$ is small, this could be achieved, by setting $\hat{v}_{\Theta}$ equal to $R^{\prime} \dot{p}_{d}-k_{e_{\Theta}} \mathbf{M}_{\Theta}^{-1} e$, for some positive constant $k_{e_{\Theta}}$. To accomplish this we introduce the error variable

$$
z_{1_{\Theta}}:=\hat{v}_{\Theta}-R^{\prime} \dot{p}_{d}+k_{e \Theta} \mathbf{M}_{\Theta}^{-1} e
$$

that we would like to drive to zero, and re-write (4) as

$$
\dot{V}_{1}=-k_{e_{\Theta}} e^{\prime} \mathbf{M}_{\Theta}^{-1} e+e^{\prime} z_{1 \Theta}-e^{\prime} \tilde{v}_{\Theta}
$$


Step 3. Backstepping for $z_{1}$ : After straightforward algebraic manipulations, the dynamic equation of the error $z_{1_{\Theta}}$ can be written as

$$
\begin{aligned}
\mathbf{M}_{\Theta} \dot{z}_{1_{\Theta}}= & S\left(\mathbf{M}_{\Theta} z_{1_{\Theta}}\right) \omega+\Gamma_{\Theta}\left(R, \hat{v}_{\Theta}, \dot{p}_{d}\right) \omega+g_{1} u_{1} \\
& +h_{1_{\Theta}}\left(e, R, \hat{v}_{\Theta}, z_{1_{\Theta}}, \ddot{p}_{d}\right)+h_{2_{\Theta}}\left(\tilde{v}_{\Theta}, \hat{v}_{\Theta}\right)
\end{aligned}
$$

where

$$
\begin{aligned}
\Gamma_{\Theta}(\cdot) & :=S\left(\mathbf{M}_{\Theta} R^{\prime} \dot{p}_{d}\right)-\mathbf{M}_{\Theta} S\left(R^{\prime} \dot{p}_{d}\right)+f_{v_{2_{\Theta}}}\left(\hat{v}_{\Theta}, R\right) \\
h_{1_{\Theta}}(\cdot) & :=f_{v_{1}}\left(\hat{v}_{\Theta}, R\right)-\mathbf{M}_{\Theta} R^{\prime} \ddot{p}_{d}+k_{e_{\Theta}} z_{1_{\Theta}}-k_{e_{\Theta}}^{2} \mathbf{M}_{\Theta}^{-1} e \\
h_{2_{\Theta}}(\cdot) & :=-\mathbf{M}_{\Theta} L_{v} \tilde{v}_{\Theta}-\beta_{v_{\Theta}}(\cdot) \mathbf{M}_{\Theta} \tilde{v}_{\Theta}-k_{e_{\Theta}} \tilde{v}_{\Theta}+S(\omega) \mathbf{M}_{\Theta} \tilde{v}_{\Theta} \\
& +f_{v_{1_{\Theta}}}(v, R)-f_{v_{1_{\Theta}}}\left(\hat{v}_{\Theta}, R\right)+\left[f_{v_{2} \Theta}(v, R)-f_{v_{2_{\Theta}}}\left(\hat{v}_{\Theta}, R\right)\right] \omega
\end{aligned}
$$

It turns out that it will not always be possible to drive $z_{1_{\Theta}}$ to zero. Instead, we will drive $z_{1_{\Theta}}$ to the constant $\delta$ in Assumption 2. To achieve this we define $\varphi_{\Theta}:=z_{1_{\Theta}}-\delta$ as a new error variable that we will drive to zero and consider the augmented control-Lyapunov function

$$
V_{2}:=V_{1}+\frac{1}{2} \varphi_{\Theta}^{\prime} \mathbf{M}_{\Theta}^{2} \varphi_{\Theta}=\frac{1}{2} e^{\prime} e+\frac{1}{2} \varphi_{\Theta}^{\prime} \mathbf{M}_{\Theta}^{2} \varphi_{\Theta} .
$$

The time derivative of $V_{2}$ can be written as

$$
\begin{aligned}
& \dot{V}_{2}=-k_{e_{\Theta}} e^{\prime} \mathbf{M}_{\Theta}^{-1} e+e^{\prime} \delta-e^{\prime} \tilde{v}_{\Theta}+\varphi_{\Theta}^{\prime}\left[\mathbf{M}_{\Theta} \mathcal{B}_{\Theta}\left(R, v, \dot{p}_{d}, \delta\right) \varsigma_{\Theta}\right. \\
& \left.+\mathbf{M}_{\Theta} h_{1_{\Theta}}\left(e, R, \hat{v}_{\Theta}, z_{1_{\Theta}}, \ddot{p}_{d}\right)+h_{3_{\Theta}}\left(\tilde{v}_{\Theta}, \tilde{\omega}_{\Theta}, \dot{p}_{d}, \hat{v}_{\Theta}, R\right)+e\right] \\
& \text { where }
\end{aligned}
$$

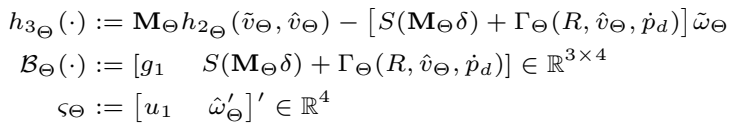

One can now regard $\varsigma_{\Theta}$ as a virtual control (actually its first component is already a "real" control) that one would like to use to make $\dot{V}_{2}$ negative. This could be achieved by setting $\varsigma_{\Theta}$ equal to ${ }^{4}$

$$
\alpha_{\Theta}:=\mathcal{B}_{\Theta}^{\prime}\left(\mathcal{B}_{\Theta} \mathcal{B}_{\Theta}^{\prime}\right)^{-1}\left(-h_{1_{\Theta}}-\mathbf{M}_{\Theta}^{-1} e-\mathbf{M}_{\Theta}^{-1} K_{\varphi_{\Theta}} \varphi_{\Theta}\right)
$$

where for each $\Theta \in \mathcal{P} K_{\varphi_{\Theta}} \in \mathbb{R}^{3 \times 3}$ is a symmetric positive definite matrix. To achieve this we set $u_{1}$ equal to the first entry of $\alpha_{\Theta}$, i.e.,

$$
u_{1}=\left[\begin{array}{ll}
1 & 0_{1 \times 3}
\end{array}\right] \alpha_{\Theta},
$$

and introduce the error variable $z_{2_{\Theta}}:=\hat{\omega}_{\Theta}-\left[\begin{array}{ll}0_{3 \times 1} I_{3 \times 3}\end{array}\right] \alpha_{\Theta}$ that one would like to set to zero. We can now re-write $\dot{V}_{2}$, with $u_{1}$ given by (7), as

$$
\begin{aligned}
\dot{V}_{2}= & -k_{e_{\Theta}} e^{\prime} \mathbf{M}_{\Theta}^{-1} e+e^{\prime} \delta-e^{\prime} \tilde{v}_{\Theta}-\varphi_{\Theta}^{\prime} K_{\varphi_{\Theta}} \varphi_{\Theta} \\
& +\varphi_{\Theta}^{\prime} \mathbf{M}_{\Theta}\left[S\left(M_{\Theta} \delta\right)+\Gamma_{\Theta}\right] z_{2_{\Theta}}+\varphi_{\Theta}^{\prime} h_{3_{\Theta}} .
\end{aligned}
$$

Step 4. Backstepping for $z_{2}$ : Consider now a the third control-Lyapunov function given by

$V_{3}:=V_{2}+\frac{1}{2} z_{2_{\Theta}}^{\prime} \mathbf{J}_{\Theta} z_{2_{\Theta}}=\frac{1}{2} e^{\prime} e+\frac{1}{2} \varphi_{\Theta}^{\prime} \mathbf{M}_{\Theta}^{2} \varphi_{\Theta}+\frac{1}{2} z_{2_{\Theta}}^{\prime} \mathbf{J}_{\Theta} z_{2_{\Theta}}$

Computing its time derivative one obtains

$$
\begin{aligned}
\dot{V}_{3}= & -k_{e_{\Theta}} e^{\prime} \mathbf{M}_{\Theta}^{-1} e+e^{\prime} \delta-e^{\prime} \tilde{v}_{\Theta}-\varphi_{\Theta}^{\prime} K_{\varphi_{\Theta}} \varphi_{\Theta}+\varphi_{\Theta}^{\prime} h_{3_{\Theta}} \\
& +z_{2_{\Theta}}^{\prime}\left[G_{2_{\Theta}} u_{2}-S(v) \mathbf{M}_{\Theta} v-S(\omega) \mathbf{J}_{\Theta} \omega+f_{\omega_{\Theta}}(v, \omega, R)\right. \\
& -\mathbf{J}_{\Theta} L_{\omega} \tilde{\omega}_{\Theta}-\beta_{\omega_{\Theta}}\left(\hat{v}_{\Theta}, R\right) \mathbf{J}_{\Theta} \tilde{\omega}_{\Theta}-\left[0_{3 \times 1} \mathbf{J}_{\Theta}\right] \dot{\alpha}_{\Theta} \\
& \left.+\left[-S\left(M_{\Theta} \delta\right)+\Gamma_{\Theta}^{\prime}\right] \mathbf{M}_{\Theta} \varphi_{\Theta}\right]
\end{aligned}
$$

${ }^{4}$ We recall that $\mathcal{B B}^{\prime}$ is nonsingular because of Assumption 2.
Selecting

$$
\begin{aligned}
u_{2}= & G_{2_{\Theta}}^{-1}\left(S(v) \mathbf{M}_{\Theta} v+S(\omega) \mathbf{J}_{\Theta} \omega-f_{\omega_{\Theta}}(v, \omega, R)\right. \\
& +\mathbf{J}_{\Theta} L_{\omega} \tilde{\omega}_{\Theta}+\beta_{\omega_{\Theta}}\left(\hat{v}_{\Theta}, R\right) \mathbf{J}_{\Theta} \tilde{\omega}_{\Theta}+\left[0_{3 \times 1} \mathbf{J}_{\Theta}\right] \dot{\alpha}_{\Theta} \\
& \left.-\left[-S\left(M_{\Theta} \delta\right)+\Gamma_{\Theta}^{\prime}\right] \mathbf{M}_{\Theta} \varphi_{\Theta}-K_{z_{2_{\Theta}}} z_{2_{\Theta}}\right),
\end{aligned}
$$

where for each $\Theta \in \mathcal{P} K_{z_{2}} \in \mathbb{R}^{3 \times 3}$ is a symmetric positive matrix, the time derivative of $V_{3}$ becomes

$$
\begin{aligned}
\dot{V}_{3}= & -k_{e_{\Theta}} e^{\prime} \mathbf{M}_{\Theta}^{-1} e-\varphi_{\Theta}^{\prime} K_{\varphi_{\Theta}} \varphi_{\Theta}-z_{2_{\Theta}}^{\prime} K_{z_{2_{\Theta}}} z_{2_{\Theta}} \\
& +e^{\prime} \delta-e^{\prime} \tilde{v}_{\Theta}+\varphi_{\Theta}^{\prime} h_{3 \Theta} .
\end{aligned}
$$

Although $\dot{V}_{3}$ has indefinite terms, they will be dominated by the negative definite terms when the estimator errors $\tilde{v}_{\Theta}, \tilde{\omega}_{\Theta}$ are sufficiently small. This is stated in the following lemma.

Lemma 2: Suppose that $u(t)=\mathcal{K}_{\Theta}(\cdot)$ for all $t \geq T^{\dagger}$, where $\mathcal{K}_{\Theta}$ is described by (7) and (8), and assume that

$$
\int_{T^{\dagger}}^{T} \gamma_{\Theta}\left(y(\tau), \tilde{v}_{\Theta}(\tau), \tilde{\omega}_{\Theta}(\tau)\right) d \tau<\infty
$$

where

$$
\begin{aligned}
\gamma_{\Theta}\left(y, \tilde{v}_{\Theta}, \tilde{\omega}_{\Theta}\right):= & \left\|\mathbf{e}_{\Theta}\right\|^{2}+\beta_{v_{\Theta}}\left(y, \tilde{v}_{\Theta}, \tilde{\omega}_{\Theta}\right)\left\|\tilde{v}_{\Theta}\right\| \\
& +\beta_{\omega_{\Theta}}\left(y, \tilde{v}_{\Theta}, \tilde{\omega}_{\Theta}\right)\left\|\tilde{\omega}_{\Theta}\right\|
\end{aligned}
$$

and $[0, T)$ denotes the maximum interval of existence of solutions to the closed-loop system. Given a sufficiently smooth time-varying desired trajectory $p_{d}:[0, T) \rightarrow \mathbb{R}^{3}$ with its time-derivatives bounded and any initial condition of the resulting closed-loop system, the signals $e(t), \hat{v}_{\Theta}(t)$, $\hat{\omega}_{\Theta}(t)$, and $u(t)$ are bounded on $\left[T^{\dagger}, T\right)$. Moreover, if $T=+\infty$, then, as $t \rightarrow \infty$, the tracking error $\left\|p(t)-p_{d}(t)\right\|$ converges to a neighborhood of the origin that can be made arbitrarily small by appropriate choice of the controller parameters.

\section{Switching-logic}

Motivated by (9)-(10), for each $\Theta \in \mathcal{P}$, we start by defining the performance signal $\mu_{\Theta}$ as the state of the dynamic equation

$$
\dot{\mu}_{\Theta}=-\lambda_{\mu} \mu_{\Theta}+\gamma_{\Theta}\left(y, \tilde{v}_{\Theta}, \tilde{\omega}_{\Theta}\right)
$$

with the initial values satisfying $\mu_{\Theta}(0)>0$. Equation (11) implies that each performance signal $\mu_{\Theta}$ is the sum of an exponentially decaying term that depends on initial conditions and a suitable exponentially weighted "norm" of the corresponding estimation errors. The control parameter $\lambda_{\mu}$ acts as a forgetting factor in the evaluation of the performance signals, hence establishing a compromise between adaptation alertness and switching dither.

The switching logic consider here is the scaleindependent hysteresis switching logic proposed in [10]. The switching signal is used to define the control signal as follows:

$$
u=\mathcal{K}_{\sigma}\left(p, R, v, \omega, \tilde{v}_{\Theta}, \tilde{\omega}_{\Theta}\right),
$$

where the candidate control laws $\mathcal{K}_{\Theta}$ are defined by (7), (8). 


\section{Stability Analysis}

Theorem 1: Given a sufficiently smooth time-varying desired trajectory $p_{d}:[0, \infty) \rightarrow \mathbb{R}^{3}$ with its time-derivatives bounded, consider the hybrid system $\Sigma_{h y b}$ described by the underactuated vehicle model (1)-(2) in closed-loop with the switched multi-controller (12), the multi-estimator (3), and the switching logic described in Section III-C.

i) For any initial condition of $\Sigma_{h y b}$ with $\mu_{\Theta}(0)>0$, $\forall \Theta \in \mathcal{P}$, the solution exists globally and all closedloop signals are bounded.

ii) Furthermore, there exists a finite time $T^{\dagger}>0$ such that $\sigma(t)=\Theta^{\dagger} \in \mathcal{P}$ for all $t \geq T^{\dagger}$ (i.e., the switching stops in finite time) and as $t \rightarrow \infty$ the tracking error $\| p(t)-$ $p_{d}(t) \|$ converges to a neighborhood of the origin that can be made arbitrarily small by appropriate choice of the control parameters.

Remark 1: The trajectory $p_{d}(t)$ can be arbitrary, that is, does not need to satisfy the dynamic model of the vehicle, and in particular can be constant for all $t \geq t_{0}$. In that case, the controller solves the position regulation problem.

Remark 2: In practice, the vector $\delta$ determines if the vehicle will follow the desired trajectory backwards or forwards: when the first component of $\delta \in \mathbb{R}^{3}$ is negative and larger (in absolute value) than the other two components, the vehicle will converge to the trajectory with positive surge velocity, and will stay "behind" the desired trajectory, see examples in Section V.

\section{Path-Following Controller Design}

In this section, the supervisory control law proposed in Section III is extended to solve the path-following problem. This extension is inspired by [18].

Let $p_{d}(\gamma) \in \mathbb{R}^{3}$ be a desired path parameterized by a continuous variable $\gamma \in \mathbb{R}$ and $v_{r}(\gamma) \in \mathbb{R}$ a desired speed assignment. Let us define the position body-fixed error $e:=$ $R^{\prime}\left[p(t)-p_{d}(\gamma(t))\right]$ and the speed error $z_{\gamma}(t):=\dot{\gamma}(t)-$ $v_{r}(\gamma(t))$. Following the same steps described in Section III$\mathrm{B}$, the time derivative of $V_{3}$ becomes

$$
\begin{aligned}
\dot{V}_{3}= & -k_{e_{\Theta}} e^{\prime} \mathbf{M}_{\Theta}^{-1} e+e^{\prime} \delta-e^{\prime} \tilde{v}_{\Theta}-\varphi_{\Theta}^{\prime} K_{\varphi_{\Theta}} \varphi_{\Theta}+\varphi_{\Theta}^{\prime} h_{3_{\Theta}} \\
& -z_{2_{\Theta}}^{\prime} K_{z_{2_{\Theta}}} z_{2_{\Theta}} \\
& +\left(-e^{\prime} R^{\prime} p_{d}^{\gamma}+\varphi^{\prime} \mathbf{M}_{\Theta} h_{4_{\Theta}}-z_{2_{\Theta}}^{\prime}\left[0_{3 \times 1} \mathbf{J}_{\Theta}\right] h_{6 \Theta}\right) z_{\gamma},
\end{aligned}
$$

where $p_{d}^{\gamma}:=\frac{\partial p_{d}}{\partial \gamma}, p_{d}^{\gamma^{2}}:=\frac{\partial^{2} p_{d}}{\partial \gamma^{2}}, v_{r}^{\gamma}:=\frac{\partial v_{r}}{\partial \gamma}, \dot{\alpha}_{\Theta}$ is decomposed as $\dot{\alpha}_{\Theta}=h_{5_{\Theta}}+h_{6 \Theta} z_{\gamma}$ and

$$
\begin{aligned}
\Gamma_{\Theta}(\cdot) & :=S\left(\mathbf{M}_{\Theta} R^{\prime} p_{d}^{\gamma} v_{r}\right)-\mathbf{M}_{\Theta} S\left(R^{\prime} p_{d}^{\gamma} v_{r}\right)+f_{v_{2_{\Theta}}}\left(\hat{v}_{\Theta}, R\right) \\
h_{1_{\Theta}}(\cdot) & :=f_{v_{1_{\Theta}}}(\cdot)-\mathbf{M}_{\Theta} R^{\prime}\left(p_{d}^{\gamma^{2}} v_{r}^{2}+p_{d}^{\gamma} v_{r}^{\gamma} v_{r}\right)+k_{e_{\Theta}} z_{1_{\Theta}}-k_{e_{\Theta}}^{2} \mathbf{M}_{\Theta}^{-1} e \\
h_{2_{\Theta}}(\cdot) & :=-\mathbf{M}_{\Theta} L_{v} \tilde{v}_{\Theta}-\beta_{1_{\Theta}}(\cdot) \mathbf{M}_{p} \tilde{v}_{\Theta}-k_{e_{\Theta}} \tilde{v}_{\Theta}+S(\omega) \mathbf{M}_{\Theta} \tilde{v}_{\Theta} \\
& +f_{v_{1}}(v, R)-f_{v_{1_{\Theta}}}\left(\hat{v}_{\Theta}, R\right)+\left[f_{v_{2_{\Theta}}}(v, R)-f_{v_{2_{\Theta}}}\left(\hat{v}_{\Theta}, R\right)\right] \omega \\
h_{4_{\Theta}}(\cdot) & :=-M_{\Theta} R^{\prime}\left(p_{d}^{\gamma^{2}} v_{r}+p_{d}^{\gamma} v_{r}^{\gamma}\right)-k_{e_{\Theta}} R^{\prime} p_{d}^{\gamma}
\end{aligned}
$$

Introduce now a forth control Lyapunov function given by

$$
V_{4}:=V_{3}+\frac{1}{2} z_{\gamma}^{2}=\frac{1}{2} e^{\prime} e+\frac{1}{2} \varphi_{\Theta}^{\prime} \mathbf{M}_{\Theta}^{2} \varphi_{\Theta}+\frac{1}{2} z_{2_{\Theta}}^{\prime} \mathbf{J}_{\Theta} z_{2_{\Theta}}+\frac{1}{2} z_{\gamma}^{2} .
$$

Computing its time derivative, we get

$$
\begin{aligned}
\dot{V}_{4}= & -k_{e_{\Theta}} e^{\prime} \mathbf{M}_{\Theta}^{-1} e+e^{\prime} \delta-e^{\prime} \tilde{v}_{\Theta}-\varphi_{\Theta}^{\prime} K_{\varphi_{\Theta}} \varphi_{\Theta}+\varphi_{\Theta}^{\prime} h_{3_{\Theta}} \\
& -z_{2_{\Theta}}^{\prime} K_{z_{\Theta}} z_{2_{\Theta}}+z_{\gamma}\left(-e^{\prime} R^{\prime} p_{d}^{\gamma}+\varphi^{\prime} \mathbf{M}_{\Theta} h_{4_{\Theta}}\right. \\
& \left.-z_{2_{\Theta}}^{\prime}\left[0_{3 \times 1} \mathbf{J}_{\Theta}\right] h_{6_{\Theta}}+\ddot{\gamma}-v_{r}^{\gamma} \dot{\gamma}\right) .
\end{aligned}
$$

Selecting the following update law for $\ddot{\gamma}$ :

$\ddot{\gamma}=e^{\prime} R^{\prime} p_{d}^{\gamma}-\varphi^{\prime} \mathbf{M}_{\Theta} h_{4_{\Theta}}+z_{2_{\Theta}}^{\prime}\left[0_{3 \times 1} \mathbf{J}_{\Theta}\right] h_{6 \Theta}+v_{r}^{\gamma} \dot{\gamma}-k_{\gamma} z_{\gamma}$,

where $k_{\gamma}$ is a positive constant, we obtain

$$
\begin{aligned}
\dot{V}_{4}= & -k_{e_{\Theta}} e^{\prime} \mathbf{M}_{\Theta}^{-1} e+e^{\prime} \delta-e^{\prime} \tilde{v}_{\Theta}-\varphi_{\Theta}^{\prime} K_{\varphi_{\Theta}} \varphi_{\Theta}+\varphi_{\Theta}^{\prime} h_{3_{\Theta}} \\
& -z_{2_{\Theta}}^{\prime} K_{z_{2_{\Theta}}} z_{2_{\Theta}}-k_{\gamma} z_{\gamma}^{2} .
\end{aligned}
$$

An extension of Theorem 1 to the path-following then follows [20].

\section{Application to Specific Vehicles}

This section illustrates the application of the previous results to two vehicles: a hovercraft (moving on a planar surface) and an underwater vehicle (moving in threedimensional space).

\section{A. Position tracking of an underactuated Hovercraft}

Consider the MVWT vehicle described in [21] consisting of a platform mounted on three low-friction, omnidirectional casters, with two attached high-performance ducted fans. Let $p=(x, y)^{\prime} \in \mathbb{R}^{2}$ be the Cartesian coordinates of the vehicle's center of mass and $\theta \in \mathbb{S}^{1}$ its orientation. Assuming that the friction and moment forces can be modeled by viscous friction, the equations of motion in the body fixed frame can be written as (1)-(2) with

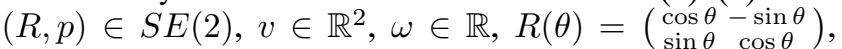
$S(\omega)=\left(\begin{array}{cc}0 & -\omega \\ \omega & 0\end{array}\right), \mathbf{M}=\operatorname{diag}\{m, m\}, \mathbf{J}=J, f_{v}=$ $-\operatorname{diag}\left\{d_{v}, d_{v}\right\} v, f_{\omega}=-d_{\omega} \omega, g_{1}=(1,0)^{\prime}, G_{2}=1$, $u_{1}=F_{s}+F_{p}, u_{2}=l\left(F_{s}-F_{p}\right)$, where $m=5.5 \mathrm{~kg}$ is the mass of the vehicle, $J=0.047 \mathrm{Kg} \mathrm{m}^{2}$ is the rotational inertia, $F_{s}$ and $F_{p}$ denote the starboard and portboard fan forces, respectively, and $l=0.123 \mathrm{~m}$ denotes the moment arm of the forces. The geometric and mass centers of the vehicle are assumed to coincide. The coefficient of viscous friction $d_{v}$ is $4.5 \mathrm{Kg} / \mathrm{s}$ and the coefficient of rotational friction $d_{\omega}$ is $0.41 \mathrm{Kg} \mathrm{m} / \mathrm{s}$. The reader is referred to [21] for a detailed coverage of the tracking controller with experimental results.

We now describe two simulation results that illustrate the performance of the proposed tracking controller with and without the supervisory control. The objective of the first experiment is to force the hovercraft to track the "virtual" kinematic unicycle vehicle

$$
\dot{x}_{d}=V_{d} \cos \theta_{d}, \quad \dot{y}_{d}=V_{d} \sin \theta_{d}, \quad \dot{\theta}_{d}=\omega_{d},
$$

which starts at $x_{d}(0)=y_{d}(0)=\theta_{d}(0)=0$ and moves with velocities $V_{d}(t)=0.2 \mathrm{~m} / \mathrm{s}$ and $\omega_{d}(t)=0.1 \mathrm{rad} / \mathrm{s}$. The initial conditions for the hovercraft are $(x, y)=$ $(-0.2 m,-1 m), R=I, v=\omega=0$. For simplicity, only the coefficient of viscous friction is unknown, but assumed to belong to the set $\mathcal{P}=\{2.5,3.0, \ldots, 6.5,7.0\}$. The control parameters were selected as follows: $k_{e_{\Theta}}=1.7$, 


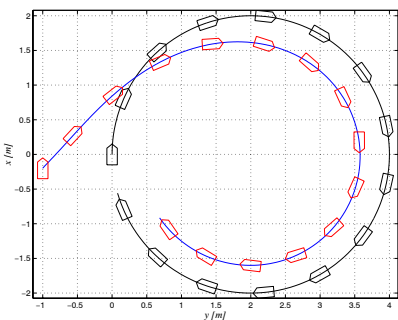

(a)

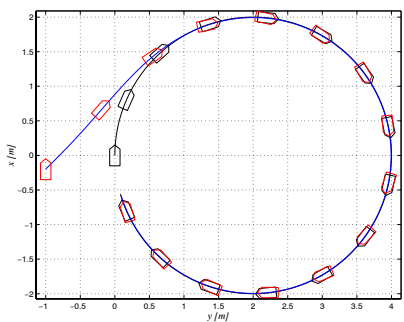

(c)

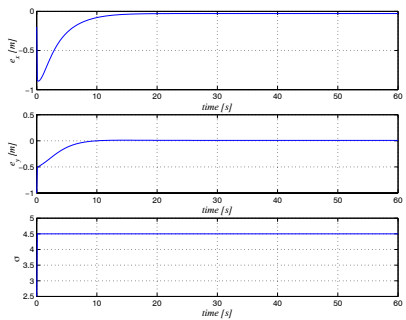

(e)

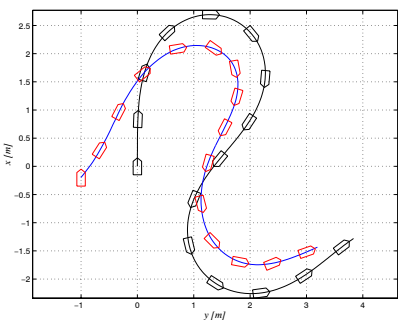

(b)

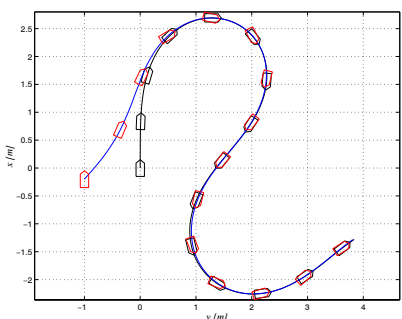

(d)

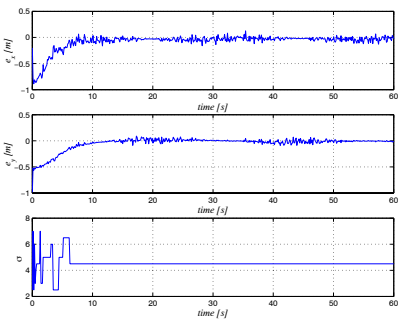

(f)
Fig. 2. Trajectory of the hovercraft in the $x y$-plane and reference trajectory performed by a unicycle vehicle using 2(a) 2(b) the position tracking controller presented in [6] and 2(c) 2(d) the estimator-based supervisory controller for trajectory-tracking. Time evolution of the tracking error in $x$ direction, in $y$-direction, and the switching signal $\sigma$ for the estimator-based supervisory controller for 2(e) the first experiment and 2(f) the second experiment.

$K_{\varphi_{\Theta}}=10 I, K_{z_{2_{\Theta}}}=10$, for all $\Theta \in \mathcal{P}$ and $\delta=$ $(-0.01,0)^{\prime}$. The hysteresis constant for the switching logic was set to $h=0.1$, the forgetting factor to $\lambda_{\mu}=1.0$, and the multi-estimator gains to $L_{v}=0.1 I$ and $L_{\omega}=0.1$. The functions $\beta_{v}(t)$ and $\beta_{\omega}(t)$ [see equation (3)] are given by $\beta_{v}(t)=\beta_{\omega}(t)=\omega^{2}$.

To illustrate the benefits derived from the supervisory control scheme proposed in Section III, we show in Fig. 2(a) the closed-loop trajectory for the (non-adaptive) trajectorytracking controller presented in [6] when the value of the coefficient of viscous friction assumed by the control system was set to $10 \%$ of the real value. It can be seen that although the closed-loop is still stable, the parameter error affects considerable the closed-loop performance. In contrast, Fig. 2(c) shows the closed-loop trajectory for the supervisory controller where, as expected, the hovercraft converges to a small neighborhood of the "virtual" unicycle vehicle, in spite of the uncertainty in $d_{v}$. Fig. 2(e) shows the time evolution of some relevant variables. Notice that in steady-state the vehicle is not aligned with the direction of the tangent velocity of $p_{d}$. Contrary to what happens for wheeled mobile robots (with inherent lateral drag coefficient $d_{v}=+\infty$ ) we cannot force the orientation $\theta$ to converge to the direction of the tangent velocity $p_{d}$.

To test the robustness of the proposed controller with respect to sensor noise, a second experiment is described. In this case, all the initial conditions and control parameters are as in the first experiment, but now the unicycle vehicle moves with velocities $V_{d}(t)=0.2 \mathrm{~m} / \mathrm{s}$ and $\omega_{d}(t)$ satisfies

$$
\dot{\omega}_{d}=-\lambda_{\omega} \omega_{d}+\lambda_{\omega} u_{\omega}, \quad \omega_{d}(0)=0
$$

where $\lambda_{\omega}=0.1$ and $u_{\omega}=0.3 \sin (t / 8)$. Zero mean uniform random noise was introduced in every sensed signal: the measured velocities $v$, and $\omega$; the orientation angle $\theta$; and the $x$ and $y$ positions. The amplitude was set to $(0.05,0.05)$, 0.05, 0.1, 0.1, and 0.1, respectively. As shown in Fig. 2(b), the hovercraft still converges to a very small neighborhood of the target unicycle vehicle.

\section{B. Trajectory-tracking and path-following of an underwater vehicle in 3-D space}

Consider an ellipsoidal shaped underactuated autonomous underwater vehicle (AUV) not necessarily neutrally buoyant. Let $\{B\}$ be a body-fixed coordinate frame whose origin is located at the center of mass of the vehicle and suppose that we have available a pure bodyfixed control force $\tau_{u}$ in the $x_{B}$ direction, and two independent control torques $\tau_{q}$ and $\tau_{r}$ about the $y_{B}$ and $z_{B}$ axes of the vehicle, respectively. The kinematics and dynamics equations of motion of the vehicle can be written as (1)-(2), where $\mathbf{M}=\operatorname{diag}\left\{m_{11}, m_{22}, m_{33}\right\}$, $\mathbf{J}=\operatorname{diag}\left\{J_{11}, J_{22}, J_{33}\right\}, u_{1}=\tau_{u}, u_{2}=\left(\tau_{q}, \tau_{r}\right)^{\prime}$, $D_{v}(v)=\operatorname{diag}\left\{X_{v_{1}}+X_{\left|v_{1}\right| v_{1}}\left|v_{1}\right|, Y_{v_{2}}+Y_{\left|v_{2}\right| v_{2}}\left|v_{2}\right|, Z_{v_{3}}+\right.$ $\left.Z_{\left|v_{3}\right| v_{3} \mid}\left|v_{3}\right|\right\}, D_{\omega}(\omega)=\operatorname{diag}\left\{K_{\omega_{1}}+K_{\left|\omega_{1}\right| \omega_{1}\left|\omega_{1}\right|, M_{\omega_{2}}+}\right.$

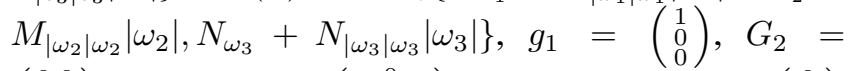
$\left(\begin{array}{ll}0 & 0 \\ 1 & 0 \\ 0 & 1\end{array}\right), \bar{g}_{1}(R)=R^{\prime}\left(\begin{array}{c}0 \\ 0 \\ W-B\end{array}\right), \bar{g}_{2}(R)=S\left(r_{B}\right) R^{\prime}\left(\begin{array}{l}0 \\ 0 \\ B\end{array}\right)$ $f_{v}(v, \omega, R)=-D_{v}(v) v-\bar{g}_{1}(R), \quad f_{\omega}(v, \omega, R)=$ $-D_{\omega}(\omega) \omega-\bar{g}_{2}(R)$. The gravitational and buoyant forces are given by $W=m g$ and $B=\rho g \nabla$, respectively, where $m$ is the mass, $\rho$ is the mass density of the water and $\nabla$ is the volume of the displaced water. The numerical values used for the physical parameters match those of the Sirene AUV, described in [22], [23].

Two simulation results are included to illustrate the dynamic behavior of the AUV in closed-loop with the trajectory-tracking controller presented in [6], and the pathfollowing controller in Section IV. Fig. 3(a) displays the vehicle trajectory using the trajectory-tracking controller in the 3D-space for the following desired trajectory $p_{d}(t)=$ $\left[V_{1} \cos \left(\frac{2 \pi}{T} t+\phi_{d}\right), V_{1} \sin \left(\frac{2 \pi}{T} t+\phi_{d}\right), V_{2} t\right]$, with $V_{1}=20$, $V_{2}=0.05, T=400$, and $\phi_{d}=-\frac{\pi}{2}$. The initial conditions of the AUV are $p=(x, y, z)=(10 m,-10 m, 0), R=I$, and $v=\omega=0$. The control parameters were selected as follows: $k_{e}=0.1, K_{\varphi}=I, K_{z_{2}}=I$, and $\delta=$ $\left(-2 \max \left(\left|m_{11}-m_{22}\right|,\left|m_{11}-m_{33}\right|\right), 0,0\right)^{\prime}$. Fig. 3(c) shows the time evolution of the Euler angles [computed from $R \in S O(3)]$. The damped oscillatory behavior of pitch and roll are due to the gravitational and buoyancy forces. Notice that the initial position of the desired position was 


\section{REFERENCES}

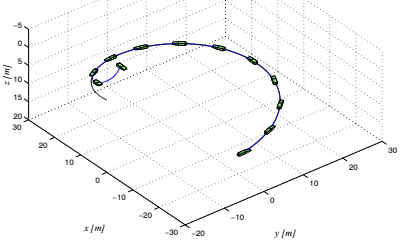

(a)

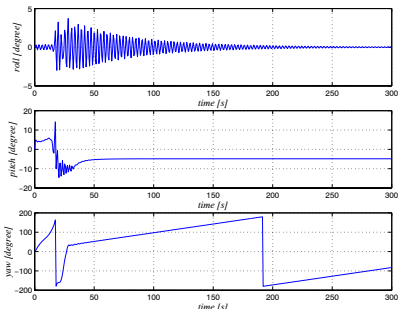

(c)

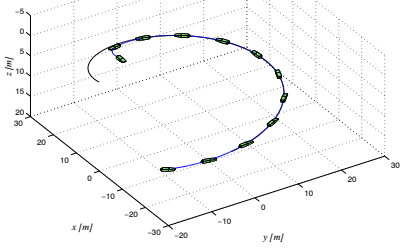

(b)

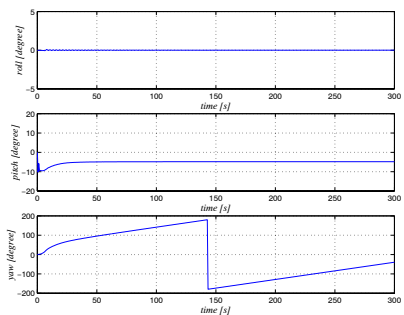

(d)
Fig. 3. Vehicle trajectory in 3-D space and time evolution of the roll $\phi$, pitch $\theta$, yaw $\psi$ Euler angles using 3(a), 3(c) the position tracking controller presented in [6]; and 3(b) 3(d) the path-following controller.

deliberately chosen to be almost behind the initial position of the vehicle. As we can see from Fig. 3(a), the vehicle turns back in its attempt to be at the given reference position at the prescribed time, requiring significant control effort and consequently inducing a strong oscillatory behavior. The path-following controller in Section IV was used to generate the trajectories in Fig. 3(b) and 3(d), where the desired path, the initial conditions and the control gains are the same as in the experiment for the tracking controller. The guidance gain and the speed assignment were set to $k_{\gamma}=2$ and $v_{r}=1 \mathrm{~m} / \mathrm{s}$, respectively. The initial condition for $\gamma$ was chosen to be the one that minimizes the distance between the initial position of the AUV and the desired path. The convergence of the vehicle to the path is now much smoother. From these two experiments one can see that when the objective is to follow a geometric path, the path-following controller offers significant performance improvement. For simplicity, in these experiments we did not include model uncertainty.

\section{CONCLUSiOnS}

We proposed a solution to the trajectory-tracking and path-following problem for underactuated autonomous vehicles in the presence of possibly large modeling parametric uncertainty. We illustrated our results in the context of two vehicle control applications: a hovercraft (moving on a planar surface) and an underwater vehicle (moving in three-dimensional space). Simulations show that the control objectives were accomplished.

Future research will address the extension of these results to a larger class of models by relaxing the requirements imposed by Assumptions 1 and 2. Another problem warrants further research is the control of underactuated vehicles with noise and in the presence of disturbances. Typical disturbances for marine vehicles include the ones induced by wave, wind, and ocean current.
[1] I. Kaminer, A. Pascoal, E. Hallberg, and C. Silvestre, "Trajectory tracking controllers for autonomous vehicles: An integrated approach to guidance and control," J. of Guidance, Control, and Dynamics, vol. 21, no. 1, pp. 29-38, 1998 .

[2] K. Y. Pettersen and H. Nijmeijer, "Underactuated ship tracking control: theory and experiments," Int. J. of Control, vol. 74, no. 14, pp. 1435-1446, 2001.

[3] Z.-P. Jiang, "Global tracking control of underactuated ships by lyapunovs direct method," Automatica, vol. 38, pp. 301-309, 2002.

[4] A. Behal, D. Dawson, W. Dixon, and Y. Fang, "Tracking and regulation control of an underactuated surface vessel with nonintegrable dynamics," IEEE Trans. on Automat. Contr., vol. 47, no. 3, pp. 495500, Mar. 2002.

[5] K. D. Do, Z. P. Jiang, and J. Pan, "Underactuated ship global tracking under relaxed conditions," IEEE Trans. on Automat. Contr., vol. 47, no. 9, pp. 1529-1536, Sept. 2002.

[6] A. P. Aguiar and J. P. Hespanha, "Position tracking of underactuated vehicles," in Proc. of the 2003 Amer. Contr. Conf., Denver, CO, USA, June 2003.

[7] A. S. Morse, "Supervisory control of families of linear set-point controllers, part 1: Exact matching," IEEE Trans. on Automat. Contr., vol. 41, no. 10, pp. 1413-1431, 1996.

[8] J. P. Hespanha and A. S. Morse, "Certainty equivalence implies detectability," Syst. \& Contr. Lett., vol. 36, no. 1, pp. 1-13, 1999.

[9] J. P. Hespanha, D. Liberzon, and A. S. Morse, "Logic-based switching control of a nonholonomic system with parametric modeling uncertainty," Syst. \& Contr. Lett., vol. 38, no. 3, pp. 167-177, 1999.

[10] — "Supervision of integral-input-to-state stabilizing controllers," Automatica, vol. 38, no. 8, pp. 1327-1335, 2002.

[11] G. Chang, J. Hespanha, A. S. Morse, M. Netto, and R. Ortega, "Supervisory field-oriented control of induction motors with uncertain rotor resistance," Int. J. of Adapt. Control and Signal Process., vol. 15 , no. 3, pp. 353-375, 2001.

[12] C. Samson, "Path-following and time-varying feedback stabilization of a wheeled mobile robot," in Proc. of the ICARCV 92, Singapore, 1992, pp. RO-13.1.1-RO-13.1.5.

[13] _ - "Control of chained systems: Application to path following and time-varying point-stabilization of mobile robots," IEEE Trans. on Automat. Contr., vol. 40, no. 1, pp. 64-77, 1995.

[14] Z.-P. Jiang and H. Nijmeijer, "A recursive technique for tracking control of nonholonomic systems in chained form," IEEE Trans. on Automat. Contr., vol. 44, no. 2, pp. 265-279, 1999.

[15] J. Hauser and R. Hindman, "Aggressive flight maneuvers," in Proc. of the 36th Conf. on Decision and Contr., San Diego, CA, USA, Dec. 1997, pp. 4186-4191.

[16] P. Encarnação and A. M. Pascoal, "3D path following control of autonomous underwater vehicles," in Proc. of the 39th Conf. on Decision and Contr., Sydney, Australia, Dec. 2000.

[17] S. Al-Hiddabi and N. McClamroch, "Tracking and maneuver regulation control for nonlinear nonminimum phase systems: application to flight control," IEEE Trans. on Contr. Systems Tech., vol. 10, no. 6, pp. 780-792, 2002.

[18] R. Skjetne, T. I. Fossen, and P. Kokotović, "Robust output maneuvering for a class of nonlinear systems," Automatica, vol. 40, no. 3, pp. 373-383, 2004.

[19] A. P. Aguiar, D. B. Dačić, J. P. Hespanha, and P. Kokotović, "Path-following or reference-tracking? An answer based on limits of performance," in Proc. of the 5th IFAC/EURON Symp. on Intel. Auton. Vehic., Lisbon, Portugal, July 2004, To appear.

[20] A. P. Aguiar and J. P. Hespanha, "Logic-based switching control for trajectory-tracking and path-following of underactuated autonomous vehicles with parametric modeling uncertainty," University of California, Santa Barbara, Tech. Rep., Sept. 2003.

[21] A. P. Aguiar, L. Cremean, and J. P. Hespanha, "Position tracking for a nonlinear underactuated hovercraft: Controller design and experimental results," in Proc. of the 42nd Conf. on Decision and Contr., Hawaii, USA, Dec. 2003.

[22] A. P. Aguiar and A. M. Pascoal, "Modeling and control of an autonomous underwater shuttle for the transport of benthic laboratories," in Proc. of the Oceans'97 Conf., Halifax, Nova Scotia, Canada, Oct. 1997.

[23] A. P. Aguiar, "Nonlinear motion control of nonholonomic and underactuated systems," Ph.D. dissertation, Dept. Electrical Engineering, Instituto Superior Técnico, IST, Lisbon, Portugal, 2002. 